\title{
High resolution optical imaging of infarction in intact organs
}

\author{
Manami Hara ${ }^{1}$, Nicolas Noiseux ${ }^{2}$, and Vytas P. Bindokas ${ }^{1}$ \\ ${ }^{1}$ The University of Chicago, Chicago, IL and ${ }^{2}$ Brigham and Women's Hospital and \\ Harvard Medical School, Boston, MA, USA \\ BioTechniques 39:373-376 (September 2005)
}

We describe a method to visualize green fluorescent protein (GFP)-labeled cells in intact organs through combined confocal and reflected laser light imaging. This method allows us a three-dimensional (3-D) view of specific cell types in situ. Imaging of tissues from transgenic mice in which the endothelial cells are labeled with GFP under the control of endothelialspecific tyrosine receptor kinase 2 (TIE2) shows the spatial distribution of the GFP-labeled endothelial cells in intact organs. We have used this method to examine the tissue necrosis in the intact heart and kidney resulting from myocardial and renal infarction. In myocardial infarction produced by surgically occluding the left anterior descending coronary artery, the border of the infarct was highly cellular and showed a disrupted endothelial network and scar tissue appearing as a dense layer of reflection. The induced renal infarction produced by ligating the renal artery in the pedicle showed a clear infarct border in the affected kidney. The 3-D reconstruction of specific cell types in the context of the surrounding tissues should be useful for studying the overall organization and the relationship between different structures in the intact organ in normal and disease states.

\section{INTRODUCTION}

The mouse has emerged as an important model for studying the pathophysiology of disease because of the relative ease of genetically modifying the mouse genome. This includes procedures for genetically tagging specific cells with fluorescent proteins and other reporters. Here we describe a method for studying the changes in tissue pathology that occurs in an infarction using green fluorescent protein (GFP)-labeled endothelial cells. The use of reflected laser light imaging as a useful complement to fluorescence confocal microscopy provides the spatial positioning of the cells in the context of their surrounding tissues in intact organs.

Reflected light imaging utilizes the differences in refractive index of tissues and subcellular structures to obtain information about unstained structures $(1,2)$. The ability to visualize the spatial distribution of specific cells in situ and in three dimensions will provide a better understanding of physiological and pathological phenomena.

\section{MATERIALS AND METHODS}

\section{Animals Used}

We used endothelial-specific tyrosine receptor kinase 2 (TIE2)-GFP transgenic mice (Jackson Laboratory, Bar Harbor, ME, USA) at the age of 78 weeks in our studies. The endothelial cells of these mice express GFP under the control of TIE2 promoter (3).

\section{Induction of Myocardial Infarction}

Mice were anesthetized with an intraperitoneal injection of pentobarbital sodium $(30 \mathrm{mg} / \mathrm{kg})$ and placed in a supine position on the operating table. An endotracheal tube (22-gauge angiocatheter) was introduced in the trachea under direct visualization. Ventilation was provided using a rodent volume controlled ventilator (687 series; Harvard Apparatus, Holliston, MA, USA) according to the manufacturer's recommendations. Through a left parasternal skin incision, the chest cavity was entered in the fourth intercostal space. The pericardial sac was opened, and the left anterior descending coronary artery was perma- nently ligated with and $8-0$ prolene suture (Ethicon, Somerville, NJ, USA). Myocardial branching indicated successful occlusion of the vessel. The chest was closed by suturing together the ribs, and the skin as separate layers. The animals were extubated when spontaneous respiration resumed.

\section{Assessment of Infarct Size}

Three days following myocardial infarction, the heart was excised, rinsed in phosphate-buffered saline (PBS), and sectioned transversally. Sections were incubated for $5 \mathrm{~min}$ at $37^{\circ} \mathrm{C}$ in $1 \%$ triphenyl tetrazolium chloride (SigmaAldrich, St. Louis, MO, USA) in PBS, washed three times in PBS, then photographed on both sides. Viable myocardium stained red, while the infarct appeared yellow-white (4).

\section{Induction of Renal Infarction}

The left renal pedicle was exposed using a left paramedian dorsal skin incision and opening of the left retroperitoneal space. The renal artery was identified in the pedicle, and one of the main renal arteries was permanently ligated distally using a 6-0 silk suture (Ethicon). The kidney was inspected for immediate color change indicating successful occlusion of the artery. Following surgery, the incision was closed in two layers, muscle and skin, and the animals were allowed to recover. For all surgeries, postoperative analgesia was given by subcutaneous injection of buprenorphine- $\mathrm{HCl}(0.05$ $\mathrm{mg} / \mathrm{kg}$; Sigma-Aldrich) for $48 \mathrm{~h}$. All surgical procedures were performed according to the National Institute of Health standards and the protocol was approved by the Harvard Medical Area Standing Committee on Animal Care and Use.

\section{Tissue Preparation}

Tissues were removed, fixed in $4 \%$ paraformaldehyde at $4{ }^{\circ} \mathrm{C}$ overnight, permeabilized in $1 \%$ Triton $^{\circledR} \mathrm{X}-100$ (Sigma-Aldrich) in PBS overnight at room temperature, and treated with the tissue-clearing reagent FocusClear ${ }^{\mathrm{TM}}$ (Pacgen Biopharmaceuticals, Burnaby, $\mathrm{BC}$, Canada) for 2 days at room 
temperature. The procedures involving mice were approved by the University of Chicago Institutional Animal Care and Use Committee.

\section{Immunohistochemistry}

Frozen sections were cut from heart tissue $6 \mu \mathrm{m}$ in thickness. Rat anti-mouse CD45 primary antibody (BD Biosciences, San Jose, CA, USA) was used to identify inflammatory cells, and goat anti-rat Alexa Fluor ${ }^{\circledR}$ 594 (Molecular Probes, Eugene, OR, USA) was used to detect the signals. Cardiomyocytes were identified using mouse monoclonal primary antibody to $\alpha$-sarcomeric actin (Sigma-Aldrich) along with Mouse and Mouse blocking system (MOM; Vector Laboratories, Burlingame, CA, USA) and Alexa Fluor 488-conjugated secondary antibody (Molecular Probes) to identify cardiomyocytes. Nuclei were stained with Hoechst 33258 (Molecular Probes).

\section{Confocal Laser Scanning Images}

Each specimen was placed in a glass-bottom dish, and confocal laser scanning images were captured using an Olympus FluoView ${ }^{\mathrm{TM}}$ IX70 confocal microscope (Olympus, Melville, NY, USA). The images of GFP-expressing endothelial cells were captured using blue excitation (488 nm wavelength) and a narrow emission filter bandwidth $(510-530 \mathrm{~nm})$. The surrounding tissue was simultaneously visualized by reflected laser light imaging using a red laser $(633 \mathrm{~nm})$ with no emission filter, and images were pseudocolored with red.

\section{Image Analysis}

Image analysis and postimage processing were performed using ImageJ (rsb.info.nih.gov/ij/). Threedimensional (3-D) reconstructions were created with Voxx software (5).

\section{RESULTS AND DISCUSSION}

We used TIE2-GFP transgenic mice, in which the endothelial cells were labeled with GFP, as a mouse model to capture high-resolution optical images of endothelial cells in intact organs (3). Tissues (lung, heart, and kidney) were removed, fixed in $4 \%$ paraformardehyde, permeabilized in $1 \%$ Triton X100 , and treated with the tissue-clearing reagent FocusClear. We have found that glycerol is not effective in most of the solid organs, and any method that requires alcohol dehydration quenches signals from fluorescent proteins. We used a water-soluble, proprietary formulation (FocusClear) that reportedly produces transparency sufficient for up to $1 \mathrm{~mm}$ observation depth in tissue (6). Our studies show little fluorescence loss in the various tissues optically sectioned up to $450 \mu \mathrm{m}$.

Figure 1 shows the images captured through combined fluorescent and reflected light confocal imaging. The signals from GFP-expressing endothelial cells were captured using blue excitation (488 $\mathrm{nm}$ wavelength) and a narrow emission filter bandwidth (510-530 nm) (Figure 1A, left panel). The surrounding tissue was simultaneously visualized by reflected laser light imaging using a red laser (633 nm) with no emission filter, and images were pseudocolored with red (Figure 1A, middle panel). The signals detected by this technique report the refractive index gradient of structures. Due to differences in their optical properties, some tissues function as reflectors by back-scattering laser light and forming bright objects, while structures such as vasculature and nuclei appear to be black. The tissue-clearing reagent FocusClear makes solid organs nearly transparent without affecting GFP signals. The reagent did not appreciably degrade laser reflection. The richest capillary network in the body is found in the lung within the interstitium of the interalveolar septum, and since the interstitium is very thin $(7,8)$, it appears as yellow in a merged GFP and reflection (red) image (Figure 1A, right panel). An animation was created by capturing real-time motion of the reconstructed image and is shown in Supplementary Video 1 found online at www.BioTechniques.com. We obtained spatial information of alveolar sacs in 3-D context by reconstructing a stack of images $(1.45 \mu \mathrm{m}$ steps, total $42 \mu \mathrm{m}$ in thickness; Figure $1 \mathrm{~B}$ and Supplementary Video 2) using volume rendering software, Voxx (5).

Pathological changes such as ischemic injury could also be captured with this technique as shown in Figure 2. Myocardial infarction was produced by permanent occlusion of the left anterior descending coronary artery (Figure $2 \mathrm{~A}$; the site of the suture in the anterior wall of the left ventricle is indicated by the $\mathrm{X}$ ) resulting in an infarct in the anterior part of the left ventricle. At 3 days postoperation, the necrotic tissue appears discolored (yellow-white), while viable myocardium stained with triphenyl tetrazolium chloride is reddish color in the representative thick cross-

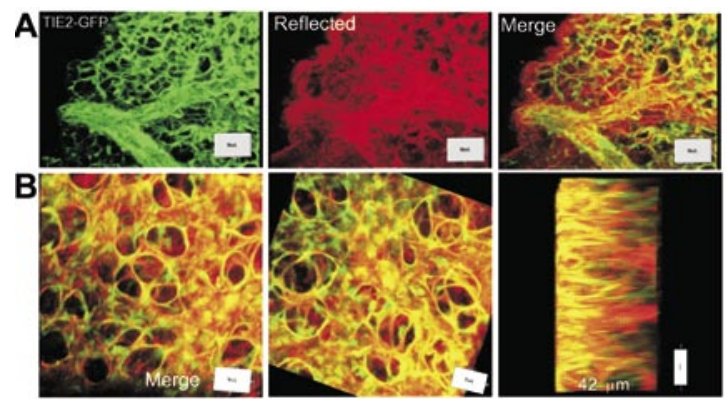

Figure 1. High resolution optical imaging of endothelial cell in the intact lung. Three-dimensional (3-D) reconstructions using a stack of confocal laser scanning images captured in the intact lung from endothelial-specific tyrosine receptor kinase 2 green fluorescent protein (TIE2-GFP) mice are shown. Each rectangular box inset shows the orientation of the view of the tissue block (bottom, bot; top; left, L; right, R; front, F; back, B). It also shows a scale in its width. (A) Lung from a TIE2-GFP mouse showing 3-D reconstruction of the respiratory tree. (A, left panel) Endothelial cells are labeled with GFP driven by the TIE2 promoter. Fluorescent image shows pulmonary capillaries that form an extensive plexus around each alveolus. (A, middle panel) Reflected light imaging showing supporting tissue which forms an attenuated layer beneath the epithelium and surrounding the blood vessels of the alveolar wall. (A, right panel) A merged image of GFP expression and reflection. Note that thin alveolar walls appear to be yellow. Orientation/scale box is 40 $\mu \mathrm{m}$ wide. 3-D reconstruction consists of 21 image planes with $1.5 \mu \mathrm{m}$ intervals (Supplementary Video 1). (B) 3-D reconstruction of the alveolar sacs in the lung. (B, left panel) A stack of merged images of fluorescence (green) and reflected light imaging (red). (B, middle panel) Rear view in the different angle shows the interconnected capillary basket surrounding each alveolus. (B, right panel) Orthogonal view of the block shows its depth of $42 \mu \mathrm{m}$. Scale is $30 \mu \mathrm{m}$. 3-D reconstruction consists of 29 image planes with $1.45 \mu \mathrm{m}$ intervals (Supplementary Video 2). 
section. On morphometric analysis, our myocardial infarction model typically resulted in infarct size of $40 \%$ of the left ventricle (data not shown). The section stained with hematoxylin and eosin (Figure 2B) shows necrotic cells and infiltration of viable myocardium by inflammatory cells forming a dense and highly cellular area at the border zone of the infarct. Many of these inflammatory cells expressing CD45, the panhematopoietic marker (in pink), infiltrated the viable and necrotic cardiomyocytes (stained in green for $\alpha$-sarcomeric actin) in the infarct border zone (Figure 2C). Nuclei were stained with Hoechst 33258 in blue and revealed the high cellular density with infiltration of mononuclear inflammatory cells in the peri-infarct area. Using reflected light and TIE2GFP fluorescence confocal imaging, a rich capillary network (Figure 2D, left panel) in the healthy myocardium
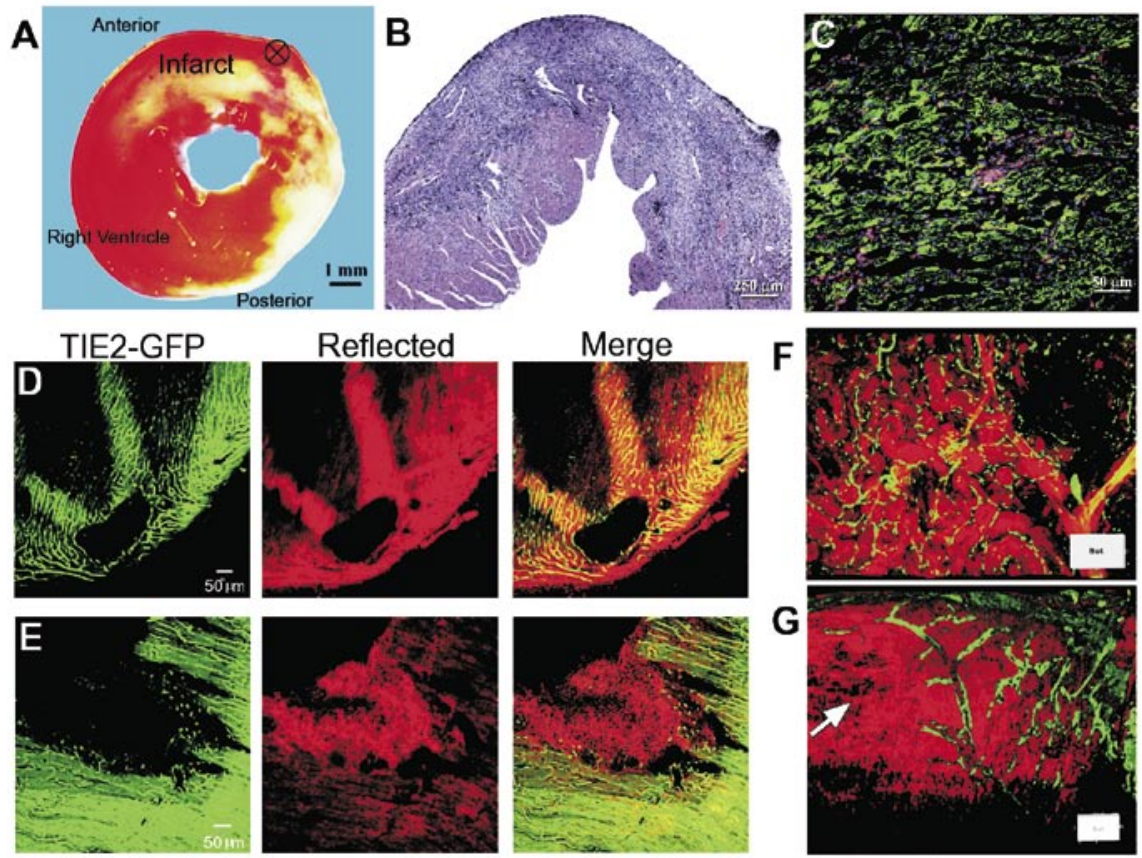

Figure 2. Pathological changes in infarcted heart and kidney 48-72 h following ischemia. (A) Myocardial infarction was produced by surgically ligating the left anterior descending coronary artery (X; the suture location in the anterior wall of the left ventricle) resulting in infarction in anterior part of heart. Representative cross-section stained with triphenyl tetrazolium chloride shows viable myocardium (red) and necrotic tissue (yellow-white). Scale bar is $1 \mathrm{~mm}$. (B) Histological section stained with hematoxylin and eosin showing infiltration of viable myocardium by inflammatory and necrotic cells forming a dense and highly cellular area at the border zone of the infarct. Scale bar is $250 \mu \mathrm{m}$. (C) Immunohistochemical staining on a frozen section showing that the panhematopoietic marker CD45 (pink) is detected in many inflammatory cells infiltrating the viable and necrotic cardiomyocytes ( $\alpha$-sarcomeric actin stained green) in the infarct border zone. Hoechst nuclear staining is shown in blue. Scale bar is $50 \mu \mathrm{m}$. (D) Healthy heart from a endothelial-specific tyrosine receptor kinase 2 green fluorescent protein (TIE2-GFP) mouse. (D, left panel) Fluorescent image shows a rich capillary network in the healthy cardiomyocyte where endothelial cells are labeled with GFP. Note that two large blood vessels merged near the apex. (D, middle panel) Reflected confocal laser light imaging showing cardiomyocytes. (D, right panel) Merged image of fluorescence and reflection. Scale bar is $50 \mu \mathrm{m}$. (E) Myocardial infarction. (E, left panel) Fluorescent image shows a cross-striated banding pattern in cardiac muscle cells. A disrupted endothelial network (GFP-expressing endothelial cells) can be seen at the border of the infarct. (E, middle panel) The scar tissue that corresponds to inflammatory cells in panel C (stained blue) appears as a dense layer of reflection in bright red. Note that the infarct area shows no reflection. (E, right panel) Merged image of fluorescence and reflection. Scale bar is $50 \mu \mathrm{m}$. (F) Kidney from a TIE2-GFP mouse. Normal kidney shows rich vascularization including glomeruli surrounded by the cortical parenchyma (reflection in red). The rectangular box inset shows the orientation of the tissue block. Scale box is $130 \mu \mathrm{m}$. Three-dimensional (3-D) reconstruction consists of 39 image planes with 3 $\mu \mathrm{m}$ intervals (Supplementary Video 3). (G) Renal infarction. Partial renal infarction shows the clear border where the endothelial network was disrupted. Scar tissue appears as a less dense layer of reflection (arrow). 3-D reconstruction consists of 45 image planes with $2 \mu \mathrm{m}$ intervals. Scale box is $125 \mu \mathrm{m}$ wide (Supplementary Video 4).
(Figure 2D, middle panel) was observed (merged in Figure 2D, right panel). Note that two large blood vessels merge near the apex. The fluorescence images show a cross-striated banding pattern in cardiac muscle cells (Figure $2 \mathrm{E}$, left panel) followed by a disrupted endothelial network at the border of the infarct. The scar tissue with infiltrating inflammatory cells appears as a dense layer of bright red reflection (Figure 2E, middle panel). Note that the infarct area composed of necrotic cardiomyocytes with disrupted normal morphology, shows low reflective property. A merged image is shown in Figure 2E, right panel.

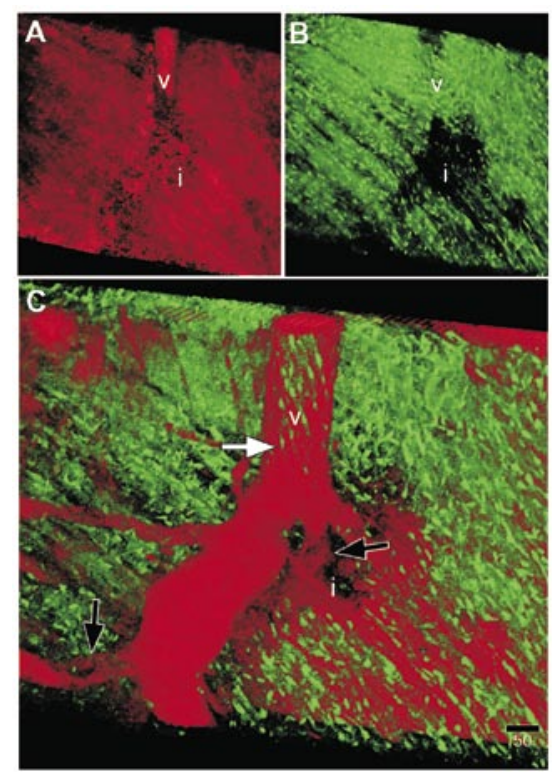

Figure 3. Detection of ischemic cardiac damage using reflected light and endothelial-specific tyrosine receptor kinase 2 green fluorescent protein (TIE2-GFP) fluorescence confocal imaging. (A) Three-dimensional (3-D) reconstruction of reflected laser light images of heart with infarct. Normal contrast shows more reflection from healthy tissue (top third) and decreased brightness in the lumen of vessel (v) and the ischemic area (i). (B) 3-D reconstruction of TIE2-GFP for the same heart tissue volume. Lettering as in panel A. Note the strand of remaining myofibrils (low-level autofluorescence) and brighter green TIE2-GFP capillaries/endothelia. (C) Magnified 3-D reconstruction of panel A (inverted contrast) and panel B. Note the vascular tissue is now bright red with prominent lateral branches. Endothelia (GFP) are absent over the large vessel (e.g., below white arrow) and the ischemic areas (i). Some lateral branches show degraded morphology or obstructions (dark arrows). See supplementary animation of the reconstructions (Supplementary Videos 5 and 6). Scale bar is $50 \mu \mathrm{m}$. 
We also examined pathological changes that occur following infarction in the kidney. In the normal kidney, the rich vascularization including glomeruli can be observed, which contrasted clearly with its surrounding cortical parenchyma by the reflected light imaging in red (Figure $2 \mathrm{~F}$ and Supplementary Video 3). Partial renal infarction produced by ligating one of the main renal arteries distally in the pedicle showed the clear disruption of the endothelial network (absent in a left part), and the scar tissue reflects strongly (Figure 2G and Supplementary Video 4). Note that the infarcted area shows low reflection as seen in myochardial infarction.

The data obtained using GFP fluorescence and reflected light confocal imaging was further processed to detect underlying ischemic cardiac damage. 3-D reconstruction of reflected laser light images of infarcted heart in normal contrast (Figure 3A) shows more reflection from an unaffected part of the tissue and decreased brightness in the lumen of vessels (v) and ischemic areas (i). 3-D reconstruction of GFP fluorescence for the same heart tissue in Figure 3B shows the endothelial cells in green. In Figure 3C, the contrast in Figure $3 \mathrm{~A}$ is inverted and merged with Figure 3B. As a result, the large blood vessel is now shown in bright red with prominent lateral branches. Endothelial cells (green) are absent over portions of the large vessel (e.g., white arrow) and ischemic areas (i). Some lateral branches show degraded morphology or obstructions (dark arrows). See also supplementary animation of the reconstruction (Supplementary Video 5 and Supplementary Video 6).

In conclusion, we describe a method for visualizing cellular detail in intact organs including pathological changes following myocardial and renal infarction without using conventional immunohistochemistry. These methods can be readily adapted to other mouse models in which specific cells and tissues are labeled with fluorescent proteins.

\section{ACKNOWLEDGMENTS}

This research was supported by U.S. Public Health Service grant nos. DK20595 and DK-61245 and Juvenile Diabetes Research Foundation to M.H. M.H. is a Naomi Berrie Fellow.

\section{COMPETING INTERESTS STATEMENT}

The authors declare no competing interests.

\section{REFERENCES}

1.Webb, R.H. and C.K. Dorey. 1995. The pixilated image, p. 55-67. In J.O. Pawley (Ed.), Handbook of Biological Confocal Microscopy, 2nd ed. Plenum Press, New York.

2.Paddock, S. 2002. Confocal reflection microscopy: the "other" confocal mode. BioTechniques 32:274-278.

3. Motoike, T., S. Loughna, E. Perens, B.L. Roman, W. Liao, T.C. Chau, C.D. Richardson, T. Kawate, et al. 2000. Universal GFP reporter for the study of vascular development. Genesis 28:75-81.

4.Melo, L.G., R. Agrawal, L. Zhang, M. Rezvani, A.A. Mangi, A. Ehsan, D.P. Griese, G. Dell'Acqua, et al. 2002. Gene therapy strategy for long-term myocardial protection using adeno-associated virus-mediated delivery of heme oxygenase gene. Circulation 105:602-607.

5.Clendenon, J.L., C.L. Phillips, R.M. Sandoval, S. Fang, and K.W. Dunn. 2002. Voxx: a PC-based, near real-time volume rendering system for biological microscopy. Am. J. Physiol. Cell Physiol. 282:C213-C218.

6. Liu, Y.C. and A.S. Chian. 2003. High-resolution confocal imaging and three-dimensional rendering. Methods 30:86-93.

7. Junqueira, L.C., J. Carneiro, and R.O. Kelley. 1998. Basic Histology, 9th ed. McGraw-Hill, New York.

8. Young, B. and J.W. Heath. 2000. Wheater's Functional Histology: A Text and Colour Atlas, 4th ed. Churchill Livingstone, Edinburgh.

Received 25 January 2005; accepted 8 April 2005.

Address correspondence to Manami Hara, Department of Medicine, The University of Chicago, 5841 South Maryland Avenue, MC1027, Chicago, IL 60637, USA. e-mail: mhara@midway.uchicago.edu

\section{To purchase reprints}

of this article, contact

apfeffer@BioTechniques.com 